\title{
Almost balancing, triangular and square triangular numbers
}

\author{
Ahmet Tekcan \\ Bursa Uludag University, Faculty of Science \\ Department of Mathematics, Bursa, Turkey \\ e-mail: tekcan@uludag.edu.tr
}

Received: 26 February 2018 Revised: 1 November 2018 Accepted: 23 February 2019

Abstract: In this work, we derive some new algebraic relations on all almost balancing numbers (of first and second type) and triangular (and also square triangular) numbers.

Keywords: Balancing numbers, Almost balancing numbers, Triangular numbers, Square triangular numbers.

2010 Mathematics Subject Classification: 11B37, 11B39.

\section{Introduction}

A positive integer $n$ is called a balancing number [1] if the Diophantine equation

$$
1+2+\cdots+(n-1)=(n+1)+(n+2)+\cdots+(n+r)
$$

holds for some positive integer $r$, which is called balancer corresponding to $n$. For example 6,35 , 204 are balancing numbers with balancers $2,14,84$, respectively.

If $n$ is a balancing number with balancer $r$, then from (1)

$$
n^{2}=\frac{(n+r)(n+r+1)}{2} \text { and } r=\frac{-2 n-1+\sqrt{8 n^{2}+1}}{2} .
$$

So from (2), $n$ is a balancing number if and only if $n^{2}$ is a triangular number (Triangular numbers denoted by $T_{n}$ are the numbers of the form

$$
T_{n}=\frac{n(n+1)}{2}
$$


for $n \geq 1$ ) and $8 n^{2}+1$ is a perfect square. Though the definition of balancing numbers suggests that no balancing number should be less than 2. Behera and Panda [1], while accepting 1 as a balancing number (since it is the positive square root of the square triangular number 1 ), have set $B_{0}=1, B_{1}=6$ and so on, using the symbol $B_{n}$ for the $n$-th balancing number. To standardize the notation at par with Fibonacci numbers, we relabel the balancing numbers by setting $B_{0}=$ $0, B_{1}=1, B_{2}=6$ and $B_{n+1}=6 B_{n}-B_{n-1}$ for $n \geq 2$.

Later Panda and Ray [13] defined that a positive integer $n$ is called a cobalancing number if the Diophantine equation

$$
1+2+\cdots+n=(n+1)+(n+2)+\cdots+(n+r)
$$

holds for some positive integer $r$, which is called cobalancer corresponding to $n$. For example 2, 14,84 are cobalancing numbers with cobalancers $1,6,35$, respectively.

If $n$ is a cobalancing number with cobalancer $r$, then from (4)

$$
n(n+1)=\frac{(n+r)(n+r+1)}{2} \text { and } r=\frac{-2 n-1+\sqrt{8 n^{2}+8 n+1}}{2} .
$$

So from (5), $n$ is a cobalancing number if and only if $n(n+1)$ is a triangular number and $8 n^{2}+8 n+1$ is a perfect square. Since $8(0)^{2}+8(0)+1=1$ is a perfect square, we accept 0 as a cobalancing number, just like Behera and Panda [1] accepted 1 as a balancing number. Cobalancing number is denoted by $b_{n}$. Then, it can be easily seen that $b_{0}=b_{1}=0, b_{2}=2$ and $b_{n+1}=6 b_{n}-b_{n-1}+2$ for $n \geq 2$.

It is clear from (1) and (4) that every balancing number is a cobalancer and every cobalancing number is a balancer, that is, $B_{n}=r_{n+1}$ and $R_{n}=b_{n}$ for $n \geq 1$, where $R_{n}$ is the $n$-th the balancer and $r_{n}$ is the $n$-th cobalancer. Since $R_{n}=b_{n}$, we get from (1) that

$$
b_{n}=\frac{-\left(2 B_{n}+1\right)+\sqrt{8 B_{n}^{2}+1}}{2} \text { and } B_{n}=\frac{2 b_{n}+1+\sqrt{8 b_{n}^{2}+8 b_{n}+1}}{2} .
$$

Thus from (6), $B_{n}$ is a balancing number if and only if $8 B_{n}^{2}+1$ is a perfect square and $b_{n}$ is a cobalancing number if and only if $8 b_{n}^{2}+8 b_{n}+1$ is a perfect square. So

$$
C_{n}=\sqrt{8 B_{n}^{2}+1} \text { and } c_{n}=\sqrt{8 b_{n}^{2}+8 b_{n}+1}
$$

are integers which are called the $n$-th Lucas-balancing number and $n$-th Lucas-cobalancing number. For example 3, 17, 99 are Lucas-balancing numbers while 1, 7, 41 are Lucas-cobalancing numbers (Note that $C_{0}=c_{0}=1$ ).

Let $\alpha=1+\sqrt{2}$ and $\beta=1-\sqrt{2}$ be the roots of the characteristic equation for Pell numbers which are the numbers defined by $P_{0}=0, P_{1}=1$ and $P_{n}=2 P_{n-1}+P_{n-2}$ for $n \geq 2$. Then Binet formulas for all balancing numbers are

$$
B_{n}=\frac{\alpha^{2 n}-\beta^{2 n}}{4 \sqrt{2}}, b_{n}=\frac{\alpha^{2 n-1}-\beta^{2 n-1}}{4 \sqrt{2}}-\frac{1}{2}, C_{n}=\frac{\alpha^{2 n}+\beta^{2 n}}{2} \text { and } c_{n}=\frac{\alpha^{2 n-1}+\beta^{2 n-1}}{2} .
$$

for $n \geq 1$ (for further details on balancing numbers see also [5, 9, 10, 14, 17, 22]). 
Balancing numbers and their generalizations have been investigated by several authors from many aspects. In [7, Theorem 4], Liptai proved that there is no Fibonacci balancing number except 1 , and in [8, Theorem 4] he proved that there is no Lucas balancing number. In [20], Szalay considered the same problem and obtained some nice results by a different method. In [6], Kovács, Liptai and Olajos extended the concept of balancing numbers to the $(a, b)$-balancing numbers defined as follows: Let $a>0$ and $b \geq 0$ be coprime integers. If

$$
(a+b)+\cdots+(a(n-1)+b)=(a(n+1)+b)+\cdots+(a(n+r)+b)
$$

for some positive integers $n$ and $r$, then $a n+b$ is an $(a, b)$-balancing number. The sequence of $(a, b)$-balancing numbers is denoted by $B_{m}^{(a, b)}$ for $m \geq 1$. In [9], Liptai, Luca, Pintér and Szalay generalized the notion of balancing numbers to numbers defined as follows: Let $y, k, l \in \mathbb{Z}^{+}$such that $y \geq 4$. Then, a positive integer $x$ with $x \leq y-2$ is called a $(k, l)$-power numerical center for $y$ if

$$
1^{k}+\cdots+(x-1)^{k}=(x+1)^{l}+\cdots+(y-1)^{l} .
$$

They studied the number of solutions of the equation above and proved several effective and ineffective finiteness results for $(k, l)$-power numerical centers.

For positive integers $k, x$, let

$$
\Pi_{k}(x)=x(x+1) \cdots(x+k-1) .
$$

It was proved in [6, Theorem 3 and Theorem 4] that the equation $B_{m}=\Pi_{k}(x)$ for fixed integer $k \geq 2$ has only infinitely many solutions and for $k \in\{2,3,4\}$ all solutions were determined. In [23, Theorem 1] Tengely, considered the case $k=5$, that is, $B_{m}=x(x+1)(x+2)(x+3)(x+4)$ and proved that this Diophantine equation has no solution for $m \geq 0$ and $x \in \mathbb{Z}$.

Recently in [3], Davala and Panda considered supercobalancing numbers and in [4], they considered the subbalancing numbers. In [2], Dash, Ota and Dash defined the $t$-balancing numbers for an integer $t \geq 2$. A positive integer $n$ is called a $t$-balancing number if the Diophantine equation

$$
1+2+\cdots+n=(n+1+t)+(n+2+t)+\cdots+(n+r+t)
$$

holds for some positive integer $r$, which is called $t$-balancer. In [21], Tekcan, Tayat and Özbek derived the general terms of $t$-balancing numbers by solving the Diophantine equation $8 x^{2}-y^{2}+$ $8 x(1+t)+(2 t+1)^{2}=0$. In [11], Özkoç and Tekcan derived some new results on $k$-balancing numbers, which are the numbers defined by $B_{0}^{k}=0, B_{1}^{k}=1, B_{n+1}^{k}=6 k B_{n}^{k}-B_{n-1}^{k}$ for $n \geq 1$, $b_{1}^{k}=0, b_{2}^{k}=2, b_{n+1}^{k}=6 k b_{n}^{k}-b_{n-1}^{k}+2$ for $n \geq 2, C_{0}^{k}=1, C_{1}^{k}=3, C_{n+1}^{k}=6 k C_{n}^{k}-C_{n-1}^{k}$ for $n \geq 1$ and $c_{1}^{k}=1, c_{2}^{k}=7, c_{n+1}^{k}=6 k c_{n}^{k}-c_{n-1}^{k}$ for $n \geq 2$, where $k$ is any positive integer (see also $[18,19])$.

Almost balancing numbers were first defined by Panda and Panda in [15]. A natural number $n$ is called an almost balancing number if the Diophantine equation

$$
|[(n+1)+(n+2)+\cdots+(n+r)]-[1+2+\cdots+(n-1)]|=1
$$

holds for some positive integer $r$, which is called the almost balancer. From (9), if $n r+\frac{r(r+1)}{2}-$ $\frac{(n-1) n}{2}=1$, then $n$ is called an almost balancing number of first type and $r$ is called an almost 
balancer of first type and in this case

$$
r=\frac{-2 n-1+\sqrt{8 n^{2}+9}}{2} .
$$

For example 3,18,105 are almost balancing numbers of first type with almost balancers of first type $1,7,43$. If $n r+\frac{r(r+1)}{2}-\frac{(n-1) n}{2}=-1$, then $n$ is called an almost balancing number of second type and $r$ is called an almost balancer of second type and in this case

$$
r=\frac{-2 n-1+\sqrt{8 n^{2}-7}}{2}
$$

For example 4,11,23 are almost balancing numbers of second type with almost balancers of second type $1,4,9$. From (11), we note that $8(1)^{2}-7=1^{2}$ and $8(2)^{2}-7=5^{2}$ are perfect squares. So we accept 1 and 2 to be almost balancing numbers of second type.

Let $B_{n}^{*}$ denote the $n$-th almost balancing number of first type and let $B_{n}^{* *}$ denote the $n$-th almost balancing number of second type. Then from (10), $B_{n}^{*}$ is an almost balancing number of first type if and only if $8\left(B_{n}^{*}\right)^{2}+9$ is a perfect square and (11), $B_{n}^{* *}$ is an almost balancing number of second type if and only if $8\left(B_{n}^{* *}\right)^{2}-7$ is a perfect square. Thus

$$
C_{n}^{*}=\sqrt{8\left(B_{n}^{*}\right)^{2}+9} \text { and } C_{n}^{* *}=\sqrt{8\left(B_{n}^{* *}\right)^{2}-7}
$$

are integers which are called the $n$-th almost Lucas-balancing number of first type and the $n$-th almost Lucas-balancing number of second type. It is easily seen that $B_{n}^{*}=3 B_{n}, C_{n}^{*}=3 C_{n}$ and $B_{2 n-1}^{* *}=B_{n-1}+C_{n-1}, B_{2 n}^{* *}=-B_{n}+C_{n}, C_{2 n-1}^{* *}=8 B_{n-1}+C_{n-1}, C_{2 n}^{* *}=8 B_{n}-C_{n}$ for $n \geq 1$ (Here we notice that $B_{0}^{*}=0, C_{0}^{*}=3, B_{0}^{* *}=1$ and $C_{0}^{* *}=-1$ ).

Similarly in [16], Panda defined that a positive integer $n$ is called an almost cobalancing number if the Diophantine equation

$$
|[(n+1)+(n+2)+\cdots+(n+r)]-(1+2+\cdots+n)|=1
$$

holds for some positive integer $r$, which is called an almost cobalancer. From (13), if $n r+$ $\frac{r(r+1)}{2}-\frac{n(n+1)}{2}=1$, then $n$ is called an almost cobalancing number of first type and $r$ is called an almost cobalancer of first type and in this case

$$
r=\frac{-2 n-1+\sqrt{8 n^{2}+8 n+9}}{2} .
$$

For example 1, 4,9 are almost cobalancing numbers of first type with almost cobalancers of first type $1,2,4$. If $n r+\frac{r(r+1)}{2}-\frac{n(n+1)}{2}=-1$, then $n$ is called an almost cobalancing number of second type and $r$ is called an almost cobalancer of second type and in this case

$$
r=\frac{-2 n-1+\sqrt{8 n^{2}+8 n-7}}{2} .
$$

For example 7,43, 253 are almost cobalancing numbers of second type with almost cobalancers of second type $3,18,105$. From (15), we note that $8(1)^{2}+8(1)-7=3^{2}$ is a perfect square. So we accept 1 as an almost cobalancing number of second type. 
Let $b_{n}^{*}$ denote the $n$-th almost cobalancing number of first type and let $b_{n}^{* *}$ denote the $n$-th almost cobalancing number of second type. Then from (14), $b_{n}^{*}$ is an almost cobalancing number of first type if and only if $8\left(b_{n}^{*}\right)^{2}+8 b_{n}^{*}+9$ is a perfect square and (15), $b_{n}^{* *}$ is an almost cobalancing number of second type if and only if $8\left(b_{n}^{* *}\right)^{2}+8 b_{n}^{* *}-7$ is a perfect square. Thus

$$
c_{n}^{*}=\sqrt{8\left(b_{n}^{*}\right)^{2}+8 b_{n}^{*}+9} \text { and } c_{n}^{* *}=\sqrt{8\left(b_{n}^{* *}\right)^{2}+8 b_{n}^{* *}-7}
$$

are integers that are called the $n$-th almost Lucas-cobalancing number of first type and the $n$-th almost Lucas-cobalancing number of second type. It is easily seen that $b_{2 n}^{*}=2 b_{n+1}-b_{n}, b_{2 n-1}^{*}=$ $4 b_{n}-b_{n-1}+1, c_{2 n}^{*}=c_{n+2}-4 c_{n+1}, c_{2 n-1}^{*}=c_{n+1}-2 c_{n}$ and $b_{n}^{* *}=3 b_{n}+1, c_{n}^{* *}=3 c_{n}$ for $n \geq 1$ (Here we notice that $b_{0}^{*}=0, c_{0}^{*}=3, b_{0}^{* *}=1$ and $c_{0}^{* *}=3$ ).

\section{Results}

In the present paper, we consider the relationship between almost balancing numbers (of first and second type) and triangular and also square triangular numbers. Recall that there are infinitely many triangular numbers that are also square numbers which are called square triangular numbers and are denoted by $S_{n}$. For example, $1,36,1225,41616, \ldots$ are square triangular numbers. For the $n$-th square triangular number $S_{n}$, we can write

$$
S_{n}=s_{n}^{2}=\frac{t_{n}\left(t_{n}+1\right)}{2}
$$

where $s_{n}$ and $t_{n}$ are the sides of the corresponding square and triangle. Binet formulas are

$$
S_{n}=\frac{\alpha^{4 n}+\beta^{4 n}-2}{32}, s_{n}=\frac{\alpha^{2 n}-\beta^{2 n}}{4 \sqrt{2}} \text { and } t_{n}=\frac{\alpha^{2 n}+\beta^{2 n}-2}{4}
$$

for $n \geq 1$. Here we accept that $S_{0}=s_{0}=t_{0}=0$ (see also [1, 12]).

Thus we can give the following results.

Theorem 2.1. For triangular numbers, we have

1. The $\left(\frac{B_{n}^{*}+B_{n-1}^{*}-3}{6}\right)$-th triangular number is $\frac{\left(b_{2 n-1}^{*}-b_{2 n-2}^{*}-1\right)\left(b_{2 n-1}^{*}-b_{2 n-2}^{*}+1\right)}{4}$, that is,

$$
\frac{T_{B_{n}^{*}+B_{n-1}^{*}-3}^{6}}{6}=\frac{\left(b_{2 n-1}^{*}-b_{2 n-2}^{*}-1\right)\left(b_{2 n-1}^{*}-b_{2 n-2}^{*}+1\right)}{4}
$$

for $n \geq 1$.

2. The $\left(\frac{7 B_{n}^{*}-B_{n-1}^{*}-3}{6}\right)$-th triangular number is $\frac{2 B_{2 n+1}^{*}+3 b_{4 n+1}^{*}-3 b_{4 n}^{*}-9}{48}$, that is,

$$
\frac{T_{7 B_{n}^{*}-B_{n-1}^{*}-3}}{6}=\frac{2 B_{2 n+1}^{*}+3 b_{4 n+1}^{*}-3 b_{4 n}^{*}-9}{48}
$$

for $n \geq 1$.

3. The $\left(\frac{B_{2 n+1}^{* *}-B_{2 n}^{* *}+B_{2 n-1}^{* *}-B_{2 n-2}^{* *}-2}{4}\right)$-th triangular number is $\frac{\left(b_{n}^{* *}-1\right)\left(b_{n}^{* *}+2\right)}{9}$, that is,

$$
\frac{T_{B_{2 n+1}^{* *}-B_{2 n}^{* *}+B_{2 n-1}^{* *}-B_{2 n-2}^{* *}-2}}{4}=\frac{\left(b_{n}^{* *}-1\right)\left(b_{n}^{* *}+2\right)}{9}
$$

for $n \geq 1$. 
4. The $\left(\frac{7 B_{2 n+1}^{* *}-7 B_{2 n}^{* *}-B_{2 n-1}^{* *}+B_{2 n-2}^{* *}-2}{4}\right)$-th triangular number is $\left(3 B_{4 n+3}^{* *}-3 B_{4 n+2}^{* *}+2 b_{2 n+1}^{* *}-8\right) /$ 48, that is,

$$
\frac{T_{7 B_{2 n+1}^{* *}-7 B_{2 n}^{* *}-B_{2 n-1}^{* *}+B_{2 n-2}^{* *}-2}}{4}=\frac{3 B_{4 n+3}^{* *}-3 B_{4 n+2}^{* *}+2 b_{2 n+1}^{* *}-8}{48}
$$

for $n \geq 1$.

Proof. (1) Applying (3), we easily deduce that

$$
\begin{aligned}
& T_{\frac{B_{n}^{*}+B_{n-1}^{*}-3}{6}}=\frac{\left(\frac{B_{n}^{*}+B_{n-1}^{*}-3}{6}\right)\left(\frac{B_{n}^{*}+B_{n-1}^{*}-3}{6}+1\right)}{2} \\
&=\frac{\left(B_{n}^{*}+B_{n-1}^{*}\right)^{2}-9}{72} \\
&=\frac{\left[3\left(\frac{\alpha^{2 n}-\beta^{2 n}}{4 \sqrt{2}}\right)+3\left(\frac{\alpha^{2 n-2}-\beta^{2 n-2}}{4 \sqrt{2}}\right)\right]^{2}-9}{72} \\
&=\frac{9\left(\alpha^{2 n}-\beta^{2 n}+\alpha^{2 n-2}-\beta^{2 n-2}\right)^{2}-288}{2304} \\
&=\frac{9\left[\alpha^{2 n-1}\left(\alpha+\alpha^{-1}\right)-\beta^{2 n-1}\left(\beta+\beta^{-1}\right)\right]^{2}-288}{2304} \\
&=\frac{9\left[2 \sqrt{2}\left(\alpha^{2 n-1}+\beta^{2 n-1}\right)\right]^{2}-288}{2304} \\
&=\frac{\alpha^{4 n-2}+\beta^{4 n-2}-6}{32} \\
&=\frac{\alpha^{4 n-2}-2(\alpha \beta)^{2 n-1}+\beta^{4 n-2}}{32}-\frac{1}{4} \\
&=\left(\frac{\alpha^{2 n-1}-\beta^{2 n-1}}{4 \sqrt{2}}\right)^{2}-\frac{1}{4} \\
&=\left(\frac{\alpha^{2 n-1}-\beta^{2 n-1}}{4 \sqrt{2}}-\frac{1}{2}\right)\left(\frac{\alpha^{2 n-1}-\beta^{2 n-1}}{4 \sqrt{2}}-\frac{1}{2}+1\right) \\
&=\frac{\left[\left(4 b_{n}-b_{n-1}+1\right)-\left(2 b_{n}-b_{n-1}\right)-1\right]\left[\left(4 b_{n}-b_{n-1}+1\right)-\left(2 b_{n}-b_{n-1}\right)+1\right]}{4} \\
&\left.=b_{2 n-2}^{*}-1\right)\left(b_{2 n-1}^{*}-b_{2 n-2}^{*}+1\right) \\
& 4
\end{aligned}
$$

The other cases can be proved similarly.

We can give the general terms of $S_{n}, s_{n}$ and $t_{n}$ in terms of almost balancing numbers of first and second type as follows:

Theorem 2.2. The general terms of $S_{n}, s_{n}$ and $t_{n}$ are

$$
\begin{aligned}
S_{n} & =\left(\frac{b_{2 n+1}^{*}-b_{2 n}^{*}-b_{2 n-1}^{*}+b_{2 n-2}^{*}}{4}\right)^{2}, n \geq 1 \\
s_{n} & =\frac{B_{n}^{*}}{3}, n \geq 1 \\
t_{n} & =\frac{C_{n}^{*}-3}{6}, n \geq 1
\end{aligned}
$$


or

$$
S_{n}=\left(\frac{b_{n+1}^{* *}-b_{n}^{* *}}{6}\right)^{2}, s_{n}=\frac{B_{2 n+1}^{* *}-B_{2 n}^{* *}}{2}, t_{n}=\frac{C_{2 n+1}^{* *}-C_{2 n}^{* *}-2}{4}
$$

for $n \geq 1$.

Proof. From (18), we get

$$
\begin{aligned}
S_{n} & =\frac{\alpha^{4 n}+\beta^{4 n}-2}{32} \\
& =\frac{\left[\alpha^{2 n}\left(\alpha-\alpha^{-1}\right)-\beta^{2 n}\left(\beta-\beta^{-1}\right)\right]^{2}}{128} \\
& =\frac{\left(\alpha^{2 n+1}-\beta^{2 n+1}-\alpha^{2 n-1}+\beta^{2 n-1}\right)^{2}}{128} \\
& =\frac{\left[\left(\frac{\alpha^{2 n+1}-\beta^{2 n+1}}{4 \sqrt{2}}-\frac{1}{2}\right)-\left(\frac{\alpha^{2 n-1}-\beta^{2 n-1}}{4 \sqrt{2}}-\frac{1}{2}\right)\right]^{2}}{4} \\
& =\frac{\left[\left(4 b_{n+1}-b_{n}+1\right)-\left(2 b_{n+1}-b_{n}\right)-\left(4 b_{n}-b_{n-1}+1\right)+\left(2 b_{n}-b_{n-1}\right)\right]^{2}}{16} \\
& =\left(\frac{\left.b_{2 n+1}^{*}-b_{2 n}^{*}-b_{2 n-1}^{*}+b_{2 n-2}^{*}\right)^{2}}{4}\right.
\end{aligned}
$$

for $n \geq 1$. Similarly we get

$$
s_{n}=\frac{\alpha^{2 n}-\beta^{2 n}}{4 \sqrt{2}}=\frac{3\left(\frac{\alpha^{2 n}-\beta^{2 n}}{4 \sqrt{2}}\right)}{3}=\frac{B_{n}^{*}}{3}
$$

and

$$
t_{n}=\frac{\alpha^{2 n}+\beta^{2 n}-2}{4}=\frac{3\left(\frac{\alpha^{2 n}+\beta^{2 n}}{2}\right)-3}{6}=\frac{C_{n}^{*}-3}{6}
$$

for $n \geq 1$. The other cases can be proved similarly.

We can also give the general terms of square triangular numbers in terms of almost balancing and almost Lucas-balancing numbers of first and second type as follows:

Theorem 2.3. The general term of $S_{n}$ is

1. $S_{n}=\left(\frac{-2 B_{n-1}^{*}+C_{n}^{*}-C_{n-1}^{*}}{6}\right)^{2}$ for $n \geq 1$.

2. $S_{n}=\frac{\left(2 B_{n-1}^{*}+C_{n-1}^{*}-3\right)\left(4 B_{n}^{*}+2 B_{n-1}^{*}+C_{n-1}^{*}+3\right)+12 B_{n}^{*}}{36}$ for $n \geq 1$.

3. $S_{n}=\left(\frac{-2 B_{2 n-1}^{* *}+2 B_{2 n-2}^{* *}+C_{2 n+1}^{* *}-C_{2 n}^{* *}-C_{2 n-1}^{* *}+C_{2 n-2}^{* *}}{4}\right)^{2}$ for $n \geq 1$.

4. $S_{n}=\left[\left(2 B_{2 n-1}^{* *}-2 B_{2 n-2}^{* *}+C_{2 n-1}^{* *}-C_{2 n-2}^{* *}-2\right)\left(4 B_{2 n+1}^{* *}-4 B_{2 n}^{* *}+2 B_{2 n-1}^{* *}-2 B_{2 n-2}^{* *}+C_{2 n-1}^{* *}-\right.\right.$ $\left.\left.C_{2 n-2}^{* *}+2\right)+8 B_{2 n+1}^{* *}-8 B_{2 n}^{* *}\right] / 16$ for $n \geq 1$.

Proof. Applying (18) yields that

$$
\begin{aligned}
S_{n} & =\frac{\alpha^{4 n}+\beta^{4 n}-2}{32} \\
& =\left(\frac{\alpha^{2 n}-\beta^{2 n}}{4 \sqrt{2}}\right)^{2}
\end{aligned}
$$




$$
\begin{aligned}
& =\left(\frac{\alpha^{2 n}\left(\frac{-3+2 \sqrt{2}}{2 \sqrt{2}}-1+\sqrt{2}\right)+\beta^{2 n}\left(\frac{3+2 \sqrt{2}}{2 \sqrt{2}}-1-\sqrt{2}\right)}{2}\right)^{2} \\
& =\left(\frac{\alpha^{2 n}\left(\frac{-2(3-2 \sqrt{2})}{4 \sqrt{2}}+\frac{1-(3-2 \sqrt{2})}{2}\right)+\beta^{2 n}\left(\frac{2(3+2 \sqrt{2})}{4 \sqrt{2}}+\frac{1-(3+2 \sqrt{2})}{2}\right)}{2}\right)^{2} \\
& =\left(\frac{\alpha^{2 n}\left(\frac{-2 \alpha^{-2}}{4 \sqrt{2}}+\frac{1-\alpha^{-2}}{2}\right)+\beta^{2 n}\left(\frac{2 \beta^{-2}}{4 \sqrt{2}}+\frac{1-\beta^{-2}}{2}\right)}{2}\right)^{2} \\
& =\left(\frac{-2\left(\frac{\alpha^{2 n-2}-\beta^{2 n-2}}{4 \sqrt{2}}\right)+\frac{\alpha^{2 n}+\beta^{2 n}}{2}-\frac{\alpha^{2 n-2}+\beta^{2 n-2}}{2}}{2}\right)^{2} \\
& =\left(\frac{\left.-2 B_{n-1}^{*}+C_{n}^{*}-C_{n-1}^{*}\right)^{2}}{6}\right.
\end{aligned}
$$

for $n \geq 1$. The other cases can be proved similarly.

Conversely, we can give the general terms of all almost balancing numbers of first and second type in terms of $s_{n}$ and $t_{n}$ as follows:

Theorem 2.4. The general terms of all almost balancing numbers of first type are

$$
\begin{aligned}
B_{n}^{*} & =3 s_{n} \\
b_{2 n}^{*} & =-2 s_{n+1}+s_{n}+2 t_{n+1}-t_{n} \\
b_{2 n-1}^{*} & =-4 s_{n}+s_{n-1}+4 t_{n}-t_{n-1}+1 \\
C_{n}^{*} & =6 t_{n}+3 \\
c_{2 n}^{*} & =3 s_{n+1}-5 s_{n} \\
c_{2 n-1}^{*} & =5 s_{n}-3 s_{n-1}
\end{aligned}
$$

for $n \geq 1$, and the general terms of all almost balancing numbers of second type are

$$
\begin{aligned}
B_{2 n}^{* *} & =-s_{n}+2 t_{n}+1 \\
B_{2 n-1}^{* *} & =s_{n-1}+2 t_{n-1}+1 \\
b_{n}^{* *} & =-3 s_{n}+3 t_{n}+1 \\
C_{2 n}^{* *} & =8 s_{n}-2 t_{n}-1 \\
C_{2 n-1}^{* *} & =8 s_{n-1}+2 t_{n-1}+1 \\
c_{n}^{* *} & =3 s_{n}+3 s_{n-1}
\end{aligned}
$$

for $n \geq 1$.

Proof. It can be proved in the same way as Theorem 2.2 and Theorem 2.3.

Thus, from Theorems 2.2 and 2.4, we construct a one-to-one correspondence between all almost balancing numbers of first and second type and $S_{n}, s_{n}, t_{n}$.

We can also give the sum of first $n$ terms of $S_{n}, s_{n}$ and $t_{n}$ in terms of almost balancing numbers of first and second type as follows: 
Theorem 2.5. The sums of first $n$-terms of $S_{n}, s_{n}$ and $t_{n}$ are

$$
\begin{aligned}
\sum_{i=1}^{n} S_{i} & =\frac{33\left(B_{n}^{*}\right)^{2}-\left(B_{n-1}^{*}\right)^{2}-18 n+9}{288} \\
\sum_{i=1}^{n} s_{i} & =\frac{5 B_{n}^{*}-B_{n-1}^{*}-3}{12} \\
\sum_{i=1}^{n} t_{i} & =\frac{7 B_{n}^{*}-B_{n-1}^{*}-6 n-3}{12}
\end{aligned}
$$

for $n \geq 1$, or

$$
\begin{aligned}
\sum_{i=1}^{n} S_{i} & =\frac{824\left(b_{n}^{* *}\right)^{2}-328 b_{n}^{* *} b_{n-1}^{* *}+660 b_{n}^{* *}+32\left(b_{n-1}^{* *}\right)^{2}-132 b_{n-1}^{* *}-72 n+168}{1152} \\
\sum_{i=1}^{n} s_{i} & =\frac{5 B_{2 n+1}^{* *}-5 B_{2 n}^{* *}-B_{2 n-1}^{* *}+B_{2 n-2}^{* *}-2}{8} \\
\sum_{i=1}^{n} t_{i} & =\frac{7 B_{2 n+1}^{* *}-7 B_{2 n}^{* *}-B_{2 n-1}^{* *}+B_{2 n-2}^{* *}-4 n-2}{8}
\end{aligned}
$$

for $n \geq 1$.

Proof. For the square triangular numbers $S_{n}$ we have $S_{n}=34 S_{n-1}-S_{n-2}+2$. Hence

$$
\begin{aligned}
S_{2} & =34 S_{1}-S_{0}+2 \\
S_{3} & =34 S_{2}-S_{1}+2 \\
& \ldots \\
S_{n-1} & =34 S_{n-2}-S_{n-3}+2 \\
S_{n} & =34 S_{n-1}-S_{n-2}+2 .
\end{aligned}
$$

If we sum both sides of (19), we obtain

$$
S_{2}+S_{3}+\cdots+S_{n}=34\left(S_{1}+S_{2}+\cdots+S_{n-1}\right)-\left(S_{0}+S_{1}+\cdots+S_{n-2}\right)+2(n-1) .
$$

Hence $-S_{1}+\left(S_{1}+S_{2}+\cdots+S_{n}\right)=34\left(S_{1}+S_{2}+\cdots+S_{n}\right)-34 S_{n}-\left[\left(S_{1}+S_{2}+\cdots+S_{n}\right)-\right.$ $\left.S_{n-1}-S_{n}\right]+2 n-2$. Since $S_{1}=1$, we get $-1+33 S_{n}-S_{n-1}-2(n-1)=32\left(S_{1}+S_{2}+\cdots+S_{n}\right)$ and hence

$$
S_{1}+S_{2}+\cdots+S_{n}=\frac{33 S_{n}-S_{n-1}-2 n+1}{32} .
$$

Note that $S_{n}=\left(\frac{b_{2 n+1}^{*}-b_{2 n}^{*}-b_{2 n-1}^{*}+b_{2 n-2}^{*}}{4}\right)^{2}$ by Theorem 2.2. We also get $b_{2 n+1}^{*}-b_{2 n}^{*}-b_{2 n-1}^{*}+$ $b_{2 n-2}^{*}=\frac{4 B_{n}^{*}}{3}$ for $n \geq 1$. So we conclude that

$$
S_{1}+S_{2}+\cdots+S_{n}=\frac{33\left(B_{n}^{*}\right)^{2}-\left(B_{n-1}^{*}\right)^{2}-18 n+9}{288}
$$

for $n \geq 1$. Again since $S_{n}=\left(\frac{b_{n+1}^{* *}-b_{n}^{* *}}{6}\right)^{2}$ by Theorem 2.2, we get

$$
S_{1}+S_{2}+\cdots+S_{n}=\frac{33\left(\frac{b_{n+1}^{* *}-b_{n}^{* *}}{6}\right)^{2}-\left(\frac{b_{n}^{* *}-b_{n-1}^{* *}}{6}\right)^{2}-2 n+1}{32}
$$




$$
=\frac{\left\{33\left(b_{n+1}^{* *}\right)^{2}-66 b_{n+1}^{* *} b_{n}^{* *}+32\left(b_{n}^{* *}\right)^{2}+2 b_{n}^{* *} b_{n-1}^{* *}-\left(b_{n-1}^{* *}\right)^{2}-72 n+36\right\}}{1152} .
$$

Taking $b_{n+1}^{* *} \rightarrow 6 b_{n}^{* *}-b_{n-1}^{* *}+2$ in (20), we get

$$
\begin{aligned}
S_{1}+S_{2}+\cdots+S_{n} & =\frac{\left\{33\left(b_{n+1}^{* *}\right)^{2}-66 b_{n+1}^{* *} b_{n}^{* *}+32\left(b_{n}^{* *}\right)^{2}+2 b_{n}^{* *} b_{n-1}^{* *}-\left(b_{n-1}^{* *}\right)^{2}-72 n+36\right\}}{1152} \\
& =\frac{824\left(b_{n}^{* *}\right)^{2}-328 b_{n}^{* *} b_{n-1}^{* *}+660 b_{n}^{* *}+32\left(b_{n-1}^{* *}\right)^{2}-132 b_{n-1}^{* *}-72 n+168}{1152}
\end{aligned}
$$

for $n \geq 1$ as we claimed. The other sums can be proved similarly.

Now we want to construct a connection between triangular and square triangular numbers. For this reason, we have to determine when the equality

$$
T_{m}=S_{n}
$$

holds for some positive integer $m$. The answer is given below.

Theorem 2.6. For the triangular and square triangular numbers, we have

1. The $\left(\frac{B_{n+1}^{*}-B_{n-1}^{*}-6}{12}\right)$-th triangular number is the $n$-th square triangular number, that is,

$$
\frac{T_{B_{n+1}^{*}-B_{n-1}^{*}-6}^{12}}{12}=S_{n}
$$

for $n \geq 1$.

2. The $\left(\frac{2 B_{n}^{*}+3 b_{2 n-1}^{*}-3 b_{2 n-2}^{*}-3}{6}\right)$-th triangular number is the $n$-th square triangular number, that is,

$$
\frac{T_{2 B_{n}^{*}+3 b_{2 n-1}^{*}-3 b_{2 n-2}^{*}-3}}{6}=S_{n}
$$

for $n \geq 1$.

3. The $\left(\frac{B_{2 n+3}^{* *}-B_{2 n+2}^{* *}-B_{2 n-1}^{* *}+B_{2 n-2}^{* *}-4}{8}\right)$-th triangular number is the $n$-th square triangular number, that is,

$$
\frac{T_{B_{2 n+3}^{* *}-B_{2 n+2}^{* *}-B_{2 n-1}^{* *}+B_{2 n-2}^{* *}}^{* *}}{8}=S_{n}
$$

for $n \geq 1$.

4. The $\left(\frac{3 B_{2 n+1}^{* *}-3 B_{2 n}^{* *}+2 b_{n}^{* *}-2}{6}\right)$-th triangular number is the $n$-th square triangular number, that is,

$$
\frac{T_{3 B_{2 n+1}^{* *}-3 B_{2 n}^{* *}+2 b_{n}^{* *-2}}}{6}=S_{n}
$$

for $n \geq 1$.

Proof. (1) From (3), we deduce that

$$
\begin{aligned}
T_{\frac{B_{n+1}^{*}-B_{n-1}^{*}-6}{12}} & =\frac{\left(\frac{B_{n+1}^{*}-B_{n-1}^{*}-6}{12}\right)\left(\frac{B_{n+1}^{*}-B_{n-1}^{*}-6}{12}+1\right)}{2} \\
& =\frac{\left(B_{n+1}^{*}-B_{n-1}^{*}\right)^{2}-36}{288}
\end{aligned}
$$




$$
\begin{aligned}
& =\frac{\left[3\left(\frac{\alpha^{2 n+2}-\beta^{2 n+2}}{4 \sqrt{2}}\right)-3\left(\frac{\alpha^{2 n-2}-\beta^{2 n-2}}{4 \sqrt{2}}\right)\right]^{2}-36}{288} \\
& =\frac{9\left[\alpha^{2 n}\left(\alpha^{2}-\alpha^{-2}\right)+\beta^{2 n}\left(-\beta^{2}+\beta^{-2}\right)\right]^{2}-1152}{9216} \\
& =\frac{9\left[4 \sqrt{2}\left(\alpha^{2 n}+\beta^{2 n}\right)\right]^{2}-1152}{9216} \\
& =\frac{288\left[\alpha^{4 n}+2(\alpha \beta)^{2 n}+\beta^{4 n}\right]-1152}{9216} \\
& =\frac{288\left(\alpha^{4 n}+\beta^{4 n}\right)-576}{9216} \\
& =\frac{\alpha^{4 n}+\beta^{4 n}-2}{32} \\
& =S_{n}
\end{aligned}
$$

by (18). The other cases can be proved similarly.

Finally for the perfect squares, we can give the following result.

Theorem 2.7. For the perfect squares, we have

1. $8\left(s_{n-1}+t_{n-1}\right)^{2}+8\left(s_{n-1}+t_{n-1}\right)+1$ is a perfect square and is

$$
\sqrt{8\left(s_{n-1}+t_{n-1}\right)^{2}+8\left(s_{n-1}+t_{n-1}\right)+1}=\frac{B_{n}^{*}+B_{n-1}^{*}}{3}
$$

for $n \geq 1$, or

$$
\sqrt{8\left(s_{n-1}+t_{n-1}\right)^{2}+8\left(s_{n-1}+t_{n-1}\right)+1}=\frac{B_{2 n+1}^{* *}-B_{2 n}^{* *}+B_{2 n-1}^{* *}-B_{2 n-2}^{* *}}{2}
$$

for $n \geq 1$.

2. $S_{n}-t_{n}-2 s_{n}\left(s_{n-1}+t_{n-1}\right)$ is a perfect square and is

$$
\sqrt{S_{n}-t_{n}-2 s_{n}\left(s_{n-1}+t_{n-1}\right)}=\frac{b_{2 n-1}^{* *}-b_{2 n-2}^{* *}-1}{2}
$$

for $n \geq 1$ or

$$
\sqrt{S_{n}-t_{n}-2 s_{n}\left(s_{n-1}+t_{n-1}\right)}=\frac{b_{n}^{* *}-1}{3}
$$

for $n \geq 1$.

3. $\left(s_{n-1}+t_{n-1}\right)^{2}+t_{n}+2 s_{n}\left(s_{n-1}+t_{n-1}\right)$ is a perfect square and is

$$
\sqrt{\left(s_{n-1}+t_{n-1}\right)^{2}+t_{n}+2 s_{n}\left(s_{n-1}+t_{n-1}\right)}=\frac{2 C_{n}^{*}-6 b_{2 n-1}^{*}+6 b_{2 n-2}^{*}}{12}
$$

for $n \geq 1$, or

$$
\sqrt{\left(s_{n-1}+t_{n-1}\right)^{2}+t_{n}+2 s_{n}\left(s_{n-1}+t_{n-1}\right)}=\frac{3 C_{2 n+1}^{* *}-3 C_{2 n}^{* *}-4 b_{n}^{* *}-2}{12}
$$

for $n \geq 1$. 
4. $\frac{s_{2 n}+s_{2 n-1}+t_{2 n-1}}{2}$ is a perfect square and is

for $n \geq 1$ or

$$
\sqrt{\frac{s_{2 n}+s_{2 n-1}+t_{2 n-1}}{2}}=\frac{2 B_{n}^{*}}{3}
$$

for $n \geq 1$.

$$
\sqrt{\frac{s_{2 n}+s_{2 n-1}+t_{2 n-1}}{2}}=B_{2 n+1}^{* *}-B_{2 n}^{* *}
$$

5. If $n$ is odd, then $t_{n}$ is a perfect square and is

$$
\sqrt{t_{n}}=\frac{c_{n}^{*}-c_{n-1}^{*}}{2}
$$

for $n \geq 1$, or

$$
\sqrt{t_{n}}=\frac{c_{\frac{n+1}{2}}^{* *}}{3}
$$

for $n \geq 1$. If $n$ is even, then $t_{n}+1$ is a perfect square and is

for $n \geq 2$, or

$$
\sqrt{t_{n}+1}=\frac{C_{\frac{n}{2}}^{*}}{3}
$$

$$
\sqrt{t_{n}+1}=\frac{C_{n+1}^{* *}-C_{n}^{* *}}{2}
$$

for $n \geq 2$.

Proof. (1) Since $s_{n}=\frac{\alpha^{2 n}-\beta^{2 n}}{4 \sqrt{2}}$ and $t_{n}=\frac{\alpha^{2 n}+\beta^{2 n}-2}{4}$ by (18), we get

$$
\begin{aligned}
& \sqrt{8\left(s_{n-1}+t_{n-1}\right)^{2}+8\left(s_{n-1}+t_{n-1}\right)+1} \\
& =\sqrt{8\left(\frac{\alpha^{2 n-2}-\beta^{2 n-2}}{4 \sqrt{2}}+\frac{\alpha^{2 n-2}+\beta^{2 n-2}-2}{4}\right)^{2}+8\left(\frac{\alpha^{2 n-2}-\beta^{2 n-2}}{4 \sqrt{2}}+\frac{\alpha^{2 n-2}+\beta^{2 n-2}-2}{4}\right)+1} \\
& =\sqrt{8\left(\frac{\alpha^{2 n-1}-\beta^{2 n-1}}{4 \sqrt{2}}-\frac{1}{2}\right)^{2}+8\left(\frac{\alpha^{2 n-1}-\beta^{2 n-1}}{4 \sqrt{2}}-\frac{1}{2}\right)+1} \\
& =\sqrt{\frac{\alpha^{4 n-2}+\beta^{4 n-2}}{4}-\frac{1}{2}} \\
& =\frac{\alpha^{2 n-1}+\beta^{2 n-1}}{2} \\
& =\frac{\alpha^{2 n-1}(2 \sqrt{2})+\beta^{2 n-1}(2 \sqrt{2})}{4 \sqrt{2}} \\
& =\frac{\alpha^{2 n-1}\left(\alpha+\alpha^{-1}\right)+\beta^{2 n-1}\left(-\beta-\beta^{-1}\right)}{4 \sqrt{2}} \\
& =\frac{\alpha^{2 n}-\beta^{2 n}}{4 \sqrt{2}+\frac{\alpha^{2 n-2}-\beta^{2 n-2}}{4 \sqrt{2}}} \\
& =\frac{3\left(\frac{\alpha^{2 n}-\beta^{2 n}}{4 \sqrt{2}}\right)+3\left(\frac{\alpha^{2 n-2}-\beta^{2 n-2}}{4 \sqrt{2}}\right)}{3} \\
& =\frac{B_{n}^{*}+B_{n-1}^{*}}{3} \\
& n \geq 1 \text { Ther }
\end{aligned}
$$

for $n \geq 1$. The other cases can be proved similarly. 


\section{Acknowledgments}

The authors wish to thank the anonymous referees for their detailed comments, carefully reading and suggestions, which have improved the presentation and quality of the paper.

\section{References}

[1] Behera, A. \& Panda, G. K. (1999). On the Square Roots of Triangular Numbers. The Fibonacci Quarterly, 37 (2), 98-105.

[2] Dash, K. K., Ota, R. S. \& Dash, S. (2012). t-Balancing Numbers. Int. J. Contemp. Math. Sciences, 7 (41), 1999-2012.

[3] Davala, R. K. \& Panda, G. K. (2016). Supercobalancing Numbers. Matematika, 32 (1), $31-42$.

[4] Davala, R. K. \& Panda, G. K. (2018). Subbalancing Numbers. Matematika, 34 (1), 163-172.

[5] Gözeri, G. K., Özkoç, A. \& Tekcan, A. (2017). Some Algebraic Relations on Balancing Numbers. Utilitas Mathematica, 103, 217-236.

[6] Kovacs, T., Liptai, K. \& Olajos, P. (2010). On (a,b)-Balancing Numbers. Publ. Math. Deb., 77 (3-4), 485-498.

[7] Liptai, K. (2004). Fibonacci Balancing Numbers. The Fibonacci Quarterly, 42 (4), 330-340.

[8] Liptai, K. (2006). Lucas Balancing Numbers. Acta Math. Univ. Ostrav., 14, $43-47$.

[9] Liptai, K., Luca, F., Pinter, A. \& Szalay, L. (2009). Generalized Balancing Numbers. Indag. Mathem., 20(1), 87-100.

[10] Olajos, P. (2010). Properties of Balancing, Cobalancing and Generalized Balancing Numbers. Annales Mathematicae et Informaticae, 37, 125-138.

[11] Özkoç, A. \& Tekcan, A. (2017). On $k$-balancing numbers. Notes on Number Theory and Discrete Maths., 23 (3), 38-52.

[12] Özkoç, A., Tekcan, A. \& Gözeri, G. K. (2017). Triangular and Square Triangular Numbers Involving Generalized Pell Numbers. Utilitas Mathematica, 102, 231-254.

[13] Panda, G. K. \& Ray, P. K. (2005). Cobalancing Numbers and Cobalancers. Int. J. Math. Math. Sci., 8, 1189-1200.

[14] Panda, G. K. \& Ray, P. K. (2011). Some Links of Balancing and Cobalancing Numbers with Pell and Associated Pell Numbers. Bul. of Inst. of Math. Acad. Sinica, 6 (1), 41-72.

[15] Panda, G. K. \& Panda, A. K. (2015). Almost Balancing Numbers. Journal of the Indian Math. Soc., 82 (3-4), 147-156. 
[16] Panda, A. K. (2017). Some Variants of the Balancing Sequences. Ph.D. thesis, National Institute of Technology Rourkela, India.

[17] Ray, P. K. (2009). Balancing and Cobalancing Numbers. Ph.D. thesis, Department of Mathematics, National Institute of Technology, Rourkela, India.

[18] Ray, P. K. (2018). On the Properties of $k$-Balancing Numbers. Ain Shams Engineering Journal, 9, 395-402.

[19] Sahu, J. (2015). k-Balancing Numbers and Pell's Equation of Higher Order. MSc thesis, Department of Mathematics, National Institute of Technology, Rourkela, India.

[20] Szalay, L. (2007). On the Resolution of Simultaneous Pell Equations. Ann. Math. Inform., $34,77-87$.

[21] Tekcan, A., Tayat, M. \& Özbek, M. E. (2014). The Diophantine Equation $8 x^{2}-y^{2}+8 x(1+$ $t)+(2 t+1)^{2}=0$ and $t$-Balancing Numbers. ISR Combinatorics, 2014, Article ID 897834.

[22] Tekcan, A., Özkoç, A. \& Özbek, M. E. (2016). Some Algebraic Relations on Integer Sequences Involving Oblong and Balancing Numbers. Ars Combinatoria, 128, 11-31.

[23] Tengely, S. (2013). Balancing Numbers which are Products of Consecutive Integers. Publ. Math. Deb., 83 (1-2), 197-205. 